\title{
Fibroblast growth factor 21 (FGF21) has a beneficial effect on carbohydrate and lipid metabolism and taste preferences in male and female mice with diet-induced obesity
}

\author{
Balybina N \\ Department of Physiology \\ Novosibirsk State University \\ Novosibirsk, Russia \\ n.balybina@nsu.ru
}

\author{
Dubinina A, Kazantseva A, Makarova E, Yakovleva T, \\ Bazhan N \\ Laboratory of Physiological Genetics \\ Institute of Cytology and Genetics SB RAS \\ Novosibirsk, Russia
}

\begin{abstract}
Obesity is a serious problem in modern society, this being the reason for intensive search for drugs to its correction. Fibroblast growth factor 21 (FGF21) is considered as a promising candidate for the development of anti-obesity drugs: it reduces adiposity and increases insulin sensitivity. Preclinical studies of the pharmacological action of FGF21 were conducted only in males. The objective of this study was to investigate the effect of FGF21 administration on taste preferences and carbohydrate and lipid metabolism in male and female mice with diet-induced obesity. Influence of FGF21 administration to obese male and female mice on body weight, glucose and lipid metabolism and choice between standard and high-fat diet was studied. It was shown that despite the significant sex differences in metabolic indices found in mice with diet-induced obesity, the administration of FGF21 had an equal beneficial effect on carbohydrate and lipid metabolism in mice of both sexes. In addition, exogenous FGF21 increased the attractiveness of a balanced standard food compared to a highfat diet in both male and female mice. These data expand the prospects for the use of FGF21 for pharmaceutical purposes.
\end{abstract}

Keywords - fibroblast growth factor, obesity, taste preferences, sex differences

\section{Introduction}

Obesity has become one of the main threats to human health. A food abundance and overeating caused by the preference for high-calorie fatty foods, along with a decrease in physical activity are the main factors contributing to the widespread prevalence of obesity. An intensive search for drugs to correct obesity is ongoing. Fibroblast growth factor 21 (FGF21) is considered as a promising candidate for the development of anti-obesity drugs. In laboratory models, monkeys, and humans, administration of FGF21 was shown to reduce body weight and improve blood lipid profile [1]. In experimental animals, FGF21 has an antidiabetic effect: it increases insulin sensitivity and lowers blood glucose [2]. Of special interest is the recently discovered ability of FGF21 to influence taste preferences: the administration of FGF21 reduces the intake of sweets and increases the intake of proteins, is of particular interest. Preclinical studies of the pharmacological action of FGF21 were conducted only in males. However, sex differences in metabolism are so pronounced that NIH recognized sex as an important biological variable that must be considered when conducting preclinical studies. The objective of this study was to investigate the effect of FGF21 administration on taste preferences and carbohydrate and lipid metabolism in male and female mice with diet-induced obesity.

\section{METHODS}

$\mathrm{C} 57 \mathrm{BL} / 6 \mathrm{~J}$ mice were housed in SPF vivarium of the Institute of Cytology and Genetics under a light regime of 13 $\mathrm{h}$ light and $11 \mathrm{~h}$ darkness, with ad libidum access to water and food. Obesity was induced by the addition of a high fat diet (HFD, D12492, protein 24.4\%, fats 34.6\%) to a standard diet (SD, protein $19 \%$, fats $3.3 \%$ ). Obese mice aged $7-8$ months were placed in the Pheno-Master cells and provided with HFD and SD. After two days of adaptation, body weight, fat and lean mass were measured in all mice and the mice were divided into control and experimental groups: for 7 days, the mice of control group received subcutaneous daily injections of phosphate-buffered saline (PBS), the mice of experimental group received recombinant mouse FGF21 (1 mg / kg) dissolved in the PBS. BW, motor activity, and intake of FHD and SD were measured daily. One day after the last injection, mouse fat and lean masses were measured again, then mice were sacrificed by decapitation and blood and liver samples were collected to analyze biochemical blood parameters and gene expression in the liver. Seven males and five females were administered with PBS, and seven males and five females were administered with FGF21.

\section{Results}

In control groups, both calorie intake (ANOVA, $\mathrm{P}<0.001$ ) and consumption of HFD (ANOVA, $\mathrm{P}<0.05$ ) were higher in females than in males, and there were no sex differences in motor activity. At the same time, the rate of obesity development was lower in females: in females, the proportion of fat was lower (ANOVA, $\mathrm{P}<0.05$ ), and the relative weight of lean mass was higher (ANOVA, $\mathrm{P}<0.05$ ) than in males. Administration of FGF21 reduced body weight (ANOVA, $\mathrm{P}<0.001$ ) and the relative proportion of fat (ANOVA, $\mathrm{P}=0.05$ ) with no effect on calorie intake in both males and females (Fig. 1a). Although FGF21 did not affect total calorie intake, it affected the choice between SD and HFD: regardless of sex, it increased the consumption of SD (ANOVA, $\mathrm{P}<0.001)$ and reduced the consumption of HFD (ANOVA, $\mathrm{P}<0.001$ ) (Fig. 1c, d). This FGF21 effect on food preference enhanced in the course of experiment (ANOVA, $\mathrm{P}<0.05$ ). In control groups, females and males differed in many hormonal and metabolic blood parameters: the blood levels of cholesterol (ANOVA, $\mathrm{P}<0.001$ ), insulin (ANOVA, $\mathrm{P}<0.001$ ) and leptin (ANOVA, $\mathrm{P}<0.001$ ) were lower, and level of adiponectin (ANOVA, $\mathrm{P}<0.001)$ was higher in females than in males. These differences can be associated with the influence of sex steroids or with different degree of 

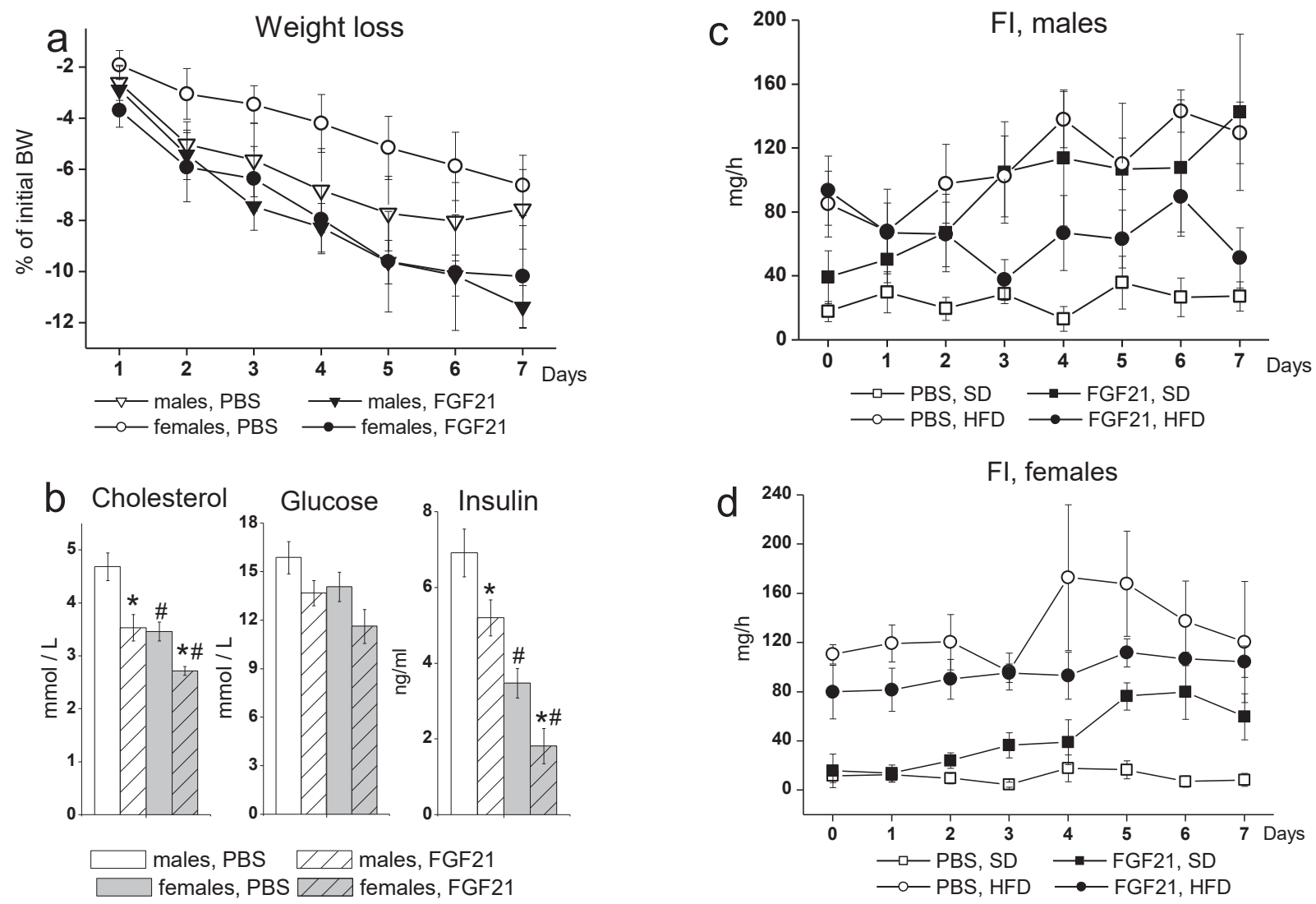

Fig. 1. Influence of FGF21 administration on body weight (a), blood biochemical characteristics (b), and consumption of standard and high-fat diet $(c, d)$ in obese male and female mice. ${ }^{*} \mathrm{P}<0.05$, PBS vs. FGF21; $\# \mathrm{P}<0.05$ males vs. females, Post Hoc Fisher LSD test.

adiposity in females and males or with both this factors. FGF21 unidirectionally affected the metabolic parameters in males and females: it lowered cholesterol (ANOVA, $\mathrm{P}<0.001$ ), glucose (ANOVA, $\mathrm{P}<0.05$ ) and insulin (ANOVA, $\mathrm{P}<0.01$ ) levels in the blood (Fig. 1b). A decrease in blood glucose and insulin levels suggests that FGF21 enhances insulin sensitivity, which is consistent with results previously obtained in males [3].

Significant sex differences in liver gene expression were observed: expressions of gene encoding insulin receptor (InsR), genes involved in the activation of fatty acid synthesis (Acca (ANOVA, P<0.001), Accb (ANOVA, $\mathrm{P}<0.05)$ ) and oxidation (Pgcla (ANOVA, $\mathrm{P}<0.05$ ), Ppara (ANOVA, $\mathrm{P}<0.05)$ ) were higher in females. Probably, the level of basal metabolism was higher in females than in males. This assumption may explain the mismatch between calorie intake and the degree of adiposity in males and females. Administration of FGF21 unidirectionally affected gene expression in the liver: it reduced the expression of genes encoding fatty acid synthase (Fasn (ANOVA, $\mathrm{P}<0.05)$ ) and pyruvate kinase (Pklr (ANOVA, $\mathrm{P}<0.05)$ ) in mice of both sexes. A decrease in the expression of these genes is possibly associated with a decrease in the level of insulin and glucose in the blood, since insulin and glucose activate the expression of Fasn in the liver [4], and glucose activates expression of the pyruvate kinase gene via activation of ChREBP. A decrease in the expression of lipogenic genes in the liver and a decrease in the consumption HFD can contribute to a decrease in blood cholesterol levels under FGF21 administration.

\section{Conclusion}

Despite the significant sex differences in metabolic indices found in mice with HFD-induced obesity, the administration of FGF21 had an equal beneficial effect on carbohydrate and lipid metabolism in mice of both sexes. In addition, exogenous FGF21 increased the attractiveness of a balanced standard food compared to a high-fat diet in both male and female mice. These data expand the prospects for the use of FGF21 for pharmaceutical purposes, since they indicate the ability of this drug to correct obesity not only via the influence on metabolic processes, but also due to mental shifts in taste preferences in favor of eating healthy balanced foods.

\section{References}

[1] V. M. Jackson, D. M. Breen, J. P. Fortin, A. Liou, J. B. Kuzmiski, A. K. Loomis et al. "Latest approaches for the treatment of obesity", Expert opinion on drug discovery, vol 10, №. 8, pp. 825-839, 2015.

[2] E. D. Berglund, C. Y. Li, H. A. Bina, S. E. Lynes, M. D. Michael, A. B. Shanafelt et al., "Fibroblast growth factor 21 controls glycemia via regulation of hepatic glucose flux and insulin sensitivity", Endocrinology, vol. 150, pp. 4084-4093, 2009.

[3] T. Coskun, H. A. Bina, M. A. Schneider, J. D. Dunbar, C. C. Hu, Y. Chen et al., "Fibroblast growth factor 21 corrects obesity in mice", vol. 149, №. 12, pp. 6018-27, 2008.

[4] Z. Song, A. M. Xiaoli, F. Yang, "Regulation and metabolic significance of de novo lipogenesis in adipose tissues”, Nutrients, vol. 10, №. 10, pp. 1383,2018 